\title{
Local time dependence of the frequency of Pi2 waves simultaneously observed at 5 low-latitude stations
}

\author{
Desheng Han $^{1}$, Toshihiko Iyemori ${ }^{2}$, Yufen $\mathrm{Gao}^{3}$, Yasuharu Sano ${ }^{4}$, Fuxi Yang ${ }^{5}$, Wansheng Li ${ }^{6}$, Masahito Nosé ${ }^{2}$ \\ ${ }^{1}$ Department of Geophysics, Graduate School of Science, Kyoto University, Kyoto 606-8502, Japan \\ ${ }^{2}$ Data Analysis Center for Geomagnetism and Space Magnetism, Graduate School of Science, Kyoto University, Kyoto 606-8502, Japan \\ ${ }^{3}$ Institute of Geophysics, CSB, Beijing 100081, China \\ ${ }^{4}$ Department of Information Management, School of Business Administration, Asahi University, Gifu 501-0296, Japan \\ ${ }^{5}$ Urumqi Basic Seismic Station Urumqi, Xinjiang Uygur Autonomous Region, 830017, China \\ ${ }^{6}$ Yinchuan Geomagnetic Observatory, Yinchuan, Ninxia Hui Autonomous Region, 750001, China
}

(Received February 27, 2003; Revised October 28, 2003; Accepted November 17, 2003)

\begin{abstract}
One-second resolution geomagnetic data from 5 stations located at low-latitudes (i.e., $L=1.2 \sim 1.6$ ) were used to examine the local time dependence of the dominant frequency of Pi2 pulsations. We analyzed $183 \mathrm{Pi} 2$ events simultaneously recorded at the 5 stations and discussed their possible generation mechanisms. The averaged dominant frequency of the $\mathrm{H}$ (horizontal) component is higher on the dawn side than that on the dusk side and shows a peak value in the post-midnight at around 03 LT, which confirms the LT dependence previously suggested from single station data (i.e., non-simultaneous observation). However, some of the events have no LT dependence. For the events which show the LT dependence at low-latitudes, we infer that the cavity resonance mode is a plausible generation mechanism, but we do not rule out the possibility of the plasmaspheric surface wave mode which has also been suggested. For the events having a common frequency between the eastern and western stations, we suggest the cavity resonance mode to be the mechanism. The averaged dominant frequency of the $\mathrm{D}$ component does not show any clear LT dependence, and only about $20 \%$ of Pi2s have identical frequency for both the H and D components, therefore we suggest that the $\mathrm{H}$ and $\mathrm{D}$ oscillations of Pi2s are generated from different mechanisms. We also found that the frequency of different parts of a Pi2 pulsation, i.e., the dominant frequency for leading part and trailing part of the pulsation, is different, and the frequency of the trailing part is lower than that of the leading part.
\end{abstract}

Key words: Pi2, frequency, LT dependence, low-latitude, simultaneous observation.

\section{Introduction}

Pi2 pulsations are impulsive, damped hydromagnetic oscillations with periods of $40-150 \mathrm{sec}$. This type of oscillations is predominantly a nighttime phenomenon and known to be associated with a substorm onset. Olson (1999) suggested that the $\mathrm{Pi} 2$ pulsations are related to two fundamental processes, a sudden generation of the field-aligned currents in association with the disruption of cross-tail currents in the plasma sheet and an impulsive response of the inner magnetosphere to the compressional waves that are generated at a substorm onset. The Pi2s observed at low and middle latitudes $(L \lesssim 4)$, where the field line is within the plasmapause, often have several discrete frequencies. These could be caused by the compressional waves that are generated at substorm onset, and when traveling inward they stimulate field line resonances and surface waves (Sutcliffe, 1975) at the plasmapause. But the most common explanation for these frequencies is that they are the resonant frequencies of the fast waves trapped within the plasmasphere, which also known as the cavity mode oscillation (Yeoman and Orr, 1989; Allan et al., 1986).

Using three ground stations at low- or mid-latitudes (i.e.,

Copy right (C) The Society of Geomagnetism and Earth, Planetary and Space Sciences (SGEPSS); The Seismological Society of Japan; The Volcanological Society of Japan The Geodetic Society of Japan; The Japanese Society for Planetary Sciences.
$L=1.2 \sim 3.4$ ), Kosaka et al. (2002) found that the frequencies of Pi2 pulsations have local time dependence, that is, they are lower on the dusk side than those on the dawn side. Two stations they used were located in the southern hemisphere and the other one is in the northern hemisphere. In their study the Pi2 pulsations were not required to be simultaneously detected at the three stations. Their results are attributed to the surface waves on the plasmapause as a plausible generation mechanism for the mid- or low-latitude Pi2 pulsations.

Lanzerotti et al. (1974) made the first attempt to use the formulation of impulsive excitation of surface modes developed by Chen and Hasegawa (1974b) to explain the Pi2 observations near the plasmapause. They were able to use the model to describe the variation of amplitudes and polarizations near the plasmapause surface and found good agreement with the data. This verified the suggestions that the surface waves could be excited in that area. By a model calculation, Kosaka et al. (2002) showed that the plasmaspheric bulge on the dusk side causes the LT dependence of the surface wave frequency. However, it was not confirmed in their study that whether "each" Pi2 event has the LT dependence or not, because the events were selected independently at each station, and there could be a bias in the event selection which could cause the apparent LT dependence. 
Table 1. Coordinates, $L$-values and time differences at the stations.

\begin{tabular}{|c|c|c|c|c|c|c|}
\hline $\begin{array}{l}\text { Station } \\
\text { name }\end{array}$ & Code & $\begin{array}{c}\text { Geographic } \\
\text { Latitude }\end{array}$ & $\begin{array}{l}\text { Geographic } \\
\text { Longitude }\end{array}$ & $\begin{array}{c}\text { Geomagnetic } \\
\text { Latitude }\end{array}$ & $L$-Value & LT-UT \\
\hline Memambetsu & MMB & 43.90 & 144.20 & 35.16 & 1.57 & $9 \mathrm{~h} 36 \mathrm{~m}$ \\
\hline Kakioka & KAK & 36.23 & 140.18 & 27.18 & 1.31 & $9 \mathrm{~h} 20 \mathrm{~m}$ \\
\hline Kanoya & KNY & 31.42 & 130.88 & 21.68 & 1.21 & $8 \mathrm{~h} 40 \mathrm{~m}$ \\
\hline Yinchuan & YCB & 38.50 & 106.30 & 28.05 & 1.42 & $7 \mathrm{~h} 4 \mathrm{~m}$ \\
\hline Urumqi & WMQ & 43.80 & 87.70 & 33.93 & 1.65 & $5 \mathrm{~h} 48 \mathrm{~m}$ \\
\hline
\end{tabular}

Local Time at Kakioka

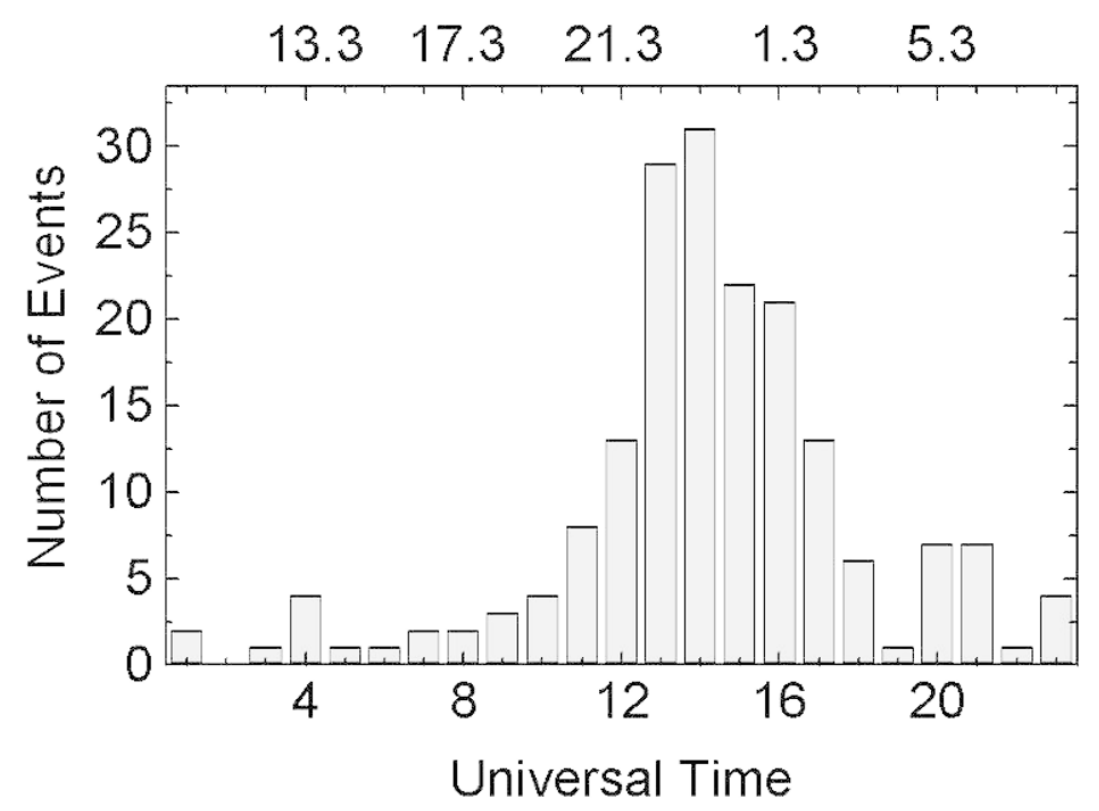

Fig. 1. Distribution of the 183 Pi2 events identified from KAK. The lower and upper frames are Universal Time (UT) and Local Time (LT) at Kakioka, respectively.

In order to confirm the validity of their results for each event, i.e., the existence of the LT dependence for each Pi2 pulsation, we identified $183 \mathrm{Pi} 2$ events commonly observed at 5 low-latitude stations which are nearly at the same latitudes but with 4 hours longitudinal difference. Then we calculated the dominant frequencies of these events, examined the frequencies as a function of LT and investigated the frequency difference between every two stations for each event. We found that the frequency of the $\mathrm{H}$ component has LT dependence, but the D component does not. On the other hand, we also found that only about $20 \%$ of the events have identical frequency for both the $\mathrm{H}$ and $\mathrm{D}$ components. We also suggest that the Pi2s observed at low-latitudes can be classified into two categories, i.e., one having LT dependence and another not having.

In Section 2, we give a description about the data set and the method of event selection. In Section 3 we present the method of analysis and results. Section 4 is the summary and discussion and Section 5 is the conclusion.

\section{Data Set and Event Selection}

One-second resolution digital data obtained at Kakioka, Kanoya, Memambetsu, Yinchuan and Urumqi were used in this study. The coordinates of these stations are given in
Table 1. The data coverage is from October 2001 to October 2002. The timing error for all of the 5 stations is smaller than 1 second in general.

The events analyzed in this study were defined from the Kakioka data. Applying an automated detection software developed by Nosé et al. (1998) to the Kakioka data, we picked up the onsets of Pi2s at first. Then, one-hour data starting 20 minutes before the onsets for all of the 5 stations were truncated out and saved into a separate file. Considering the duration of Pi2 event normally shorter than 20 minutes, if another Pi2 onset was detected within 20 minutes after the first onset, we did not create another new file to save it.

The rate of successful detection of Pi2 pulsations by the automated detection software is higher than $80 \%$ on the nightside, but less than $30 \%$ on the dayside (Nosé et al., 1998). In detection of the Pi2s by the software, we did not limit the local time to the nightside. Therefore we need to confirm that each onset detected by the software is a Pi2 but not a Pc3 or Pc4. We do this by applying a $20 \mathrm{~s}-300 \mathrm{~s}$ bandpass filter to each one-hour data which includes the detected events and plotting the filtered data to check its waveform visually. If it shows a very clear Pi2-waveform, i.e., dumped oscillation with irregular component, we determined the onset time of the event by visual inspection and truncated 5 or 

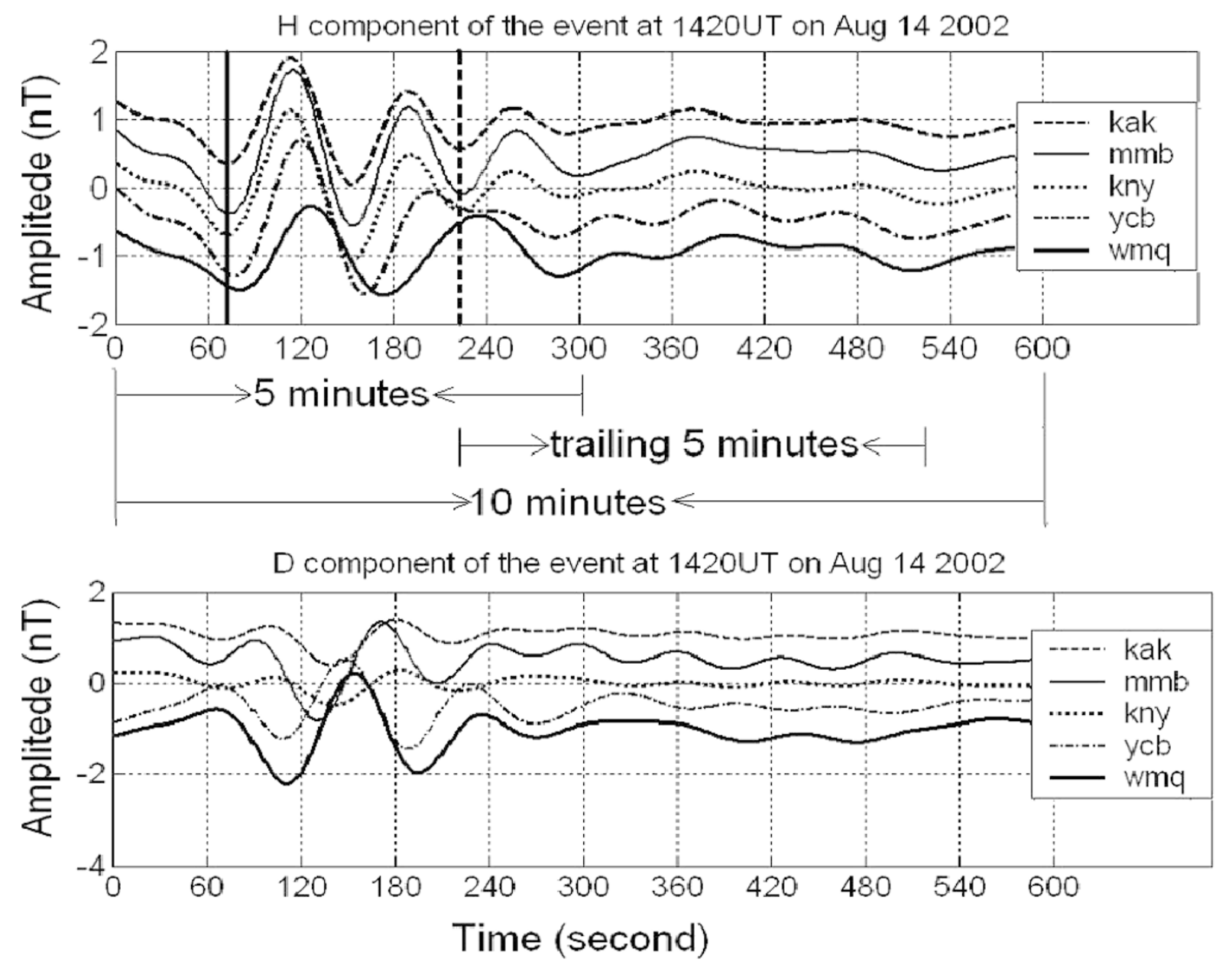

Fig. 2. Definition of the three data sets. The data sets are defined according to the waveform of the $H$ component for each event. In upper panel, the black vertical line shows the onset of the Pi2. The curves from 0 to 600 second represent an example of the 10-minute data set. For this event, the 5-minute data set is from 0 to 300 second. The trailing-5-minute data set is defined starting from 2 wavelengths after the onset and lasts 5 minutes. Here, it starts from 220 second.

10 minutes data from the original time series.

In other cases, if the waveform does not have a very clear Pi2 pattern, we referred to the LANL Geosynchronous Energetic Particle Summary Plot (http://leadbelly.lanl.gov/lanl_ep_data/) to check whether the event corresponds to a substorm onset or not. If it coincides with a substorm onset observed in the LANL summary plots, we took it as a Pi2 event and also truncated it into 5 or 10 minutes data set. With this procedure, 209 events were identified and 183 of them were observed at all of the 4 other stations. The distribution of the $183 \mathrm{Pi} 2$ events with the UT and LT at Kakioka is shown in Fig. 1.

As for defining the onset and duration of Pi2s, different criteria have been used by different authors (Takahashi et al., 1995; Cheng et al., 2000; Kosaka et al., 2002). In this paper, based on the length of the data, we adopted three types of data sets which are called "10-minute data set", "5-minute data set" and "trailing-5-minute data set", respectively. The start time of each event is defined by visual inspection according to the waveforms of the $\mathrm{H}$ component.

Figure 2 is an example of the 10-minute data set and depicts how to define the three data sets. The upper panel shows the $\mathrm{H}$ component and the lower panel is the $\mathrm{D}$ component for the same event. The solid vertical line in upper panel of
Fig. 2 indicates the onset of the Pi2 onset is selected. The start time of the 10-minute data set is taken approximately one or two minutes before the onset as shown in Fig. 2. The start time of the 5-minute data set is generally taken at somewhere between the start time of 10-minute data set and the onset of the Pi2, which is nearly the same with that used by Kosaka et al. (2002). The start time of the trailing-5-minute data set is defined as the time at 2 wavelengths after the onset, which is shown by the coarse dash line in upper panel of the Fig. 2. For some events, the waveform vanishes after 2 wave periods, so that only 136 of the 183 events, which have obvious waveforms after the main oscillations of Pi2s, were selected to examine the wave properties of the trailing-part of Pi2s.

We adopted the 5-minute data set to keep consistency in analysis method with that of Kosaka et al. (2002). Considering that there are some events which last for longer than 5 minutes, we adopted the 10-minute data set for avoiding the possible error caused by the insufficient data length to reflect the whole information of the pulsation, while the trailing-5minute data set is for investigating the possible difference in properties between different parts of Pi2 waves. 


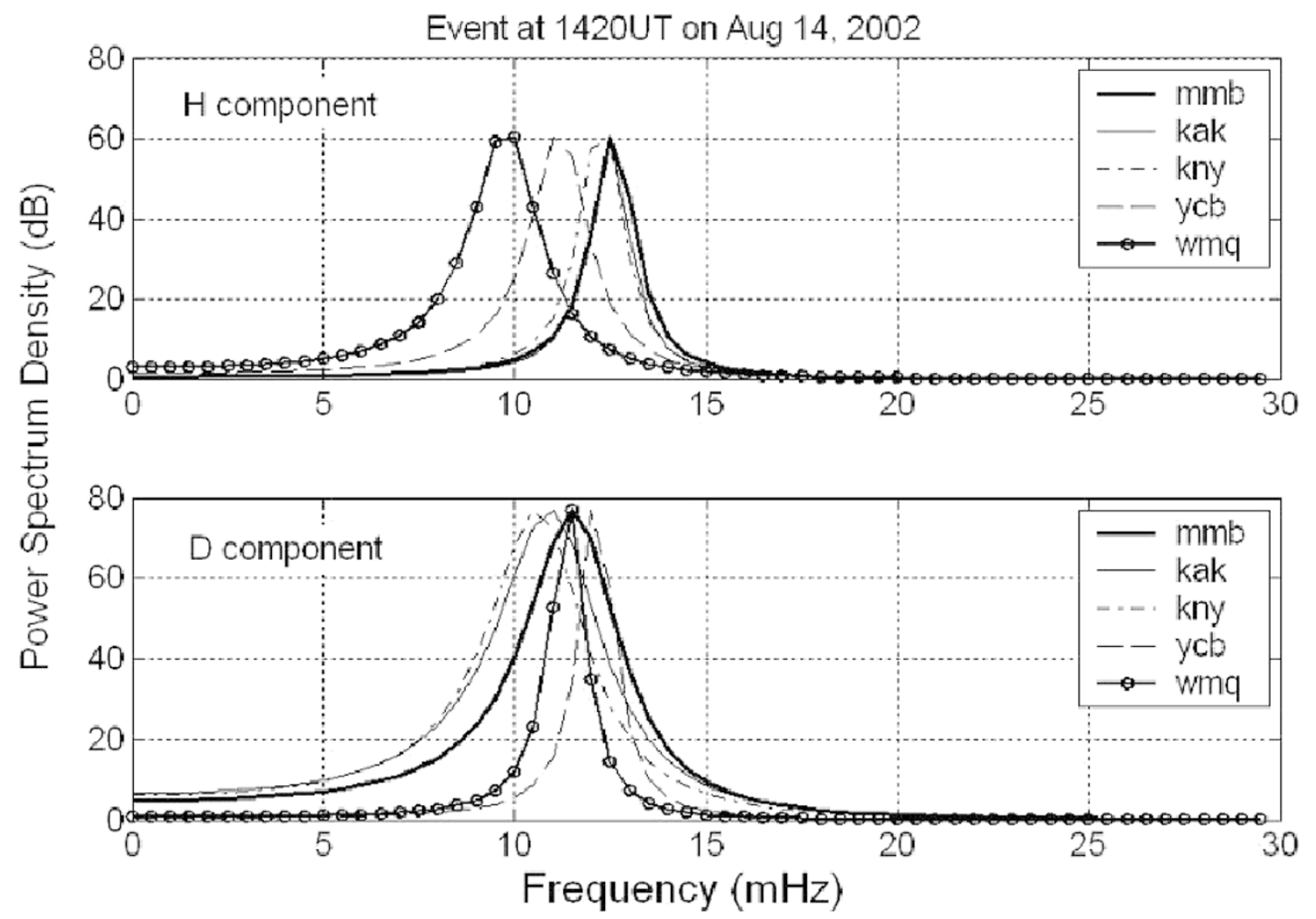

Fig. 3. An example of the MEM spectra. The results are obtained from the same event shown in Fig. 2. The frequency grid is 0.5 mHz. For the $\mathrm{H}$ component, the frequencies of the eastern stations MMB, KAK and KNY, are higher than that of the western stations YCB and WMQ, and the frequency of WMQ is the lowest. But for the D component, the frequency difference between the western and eastern stations is not so regular.

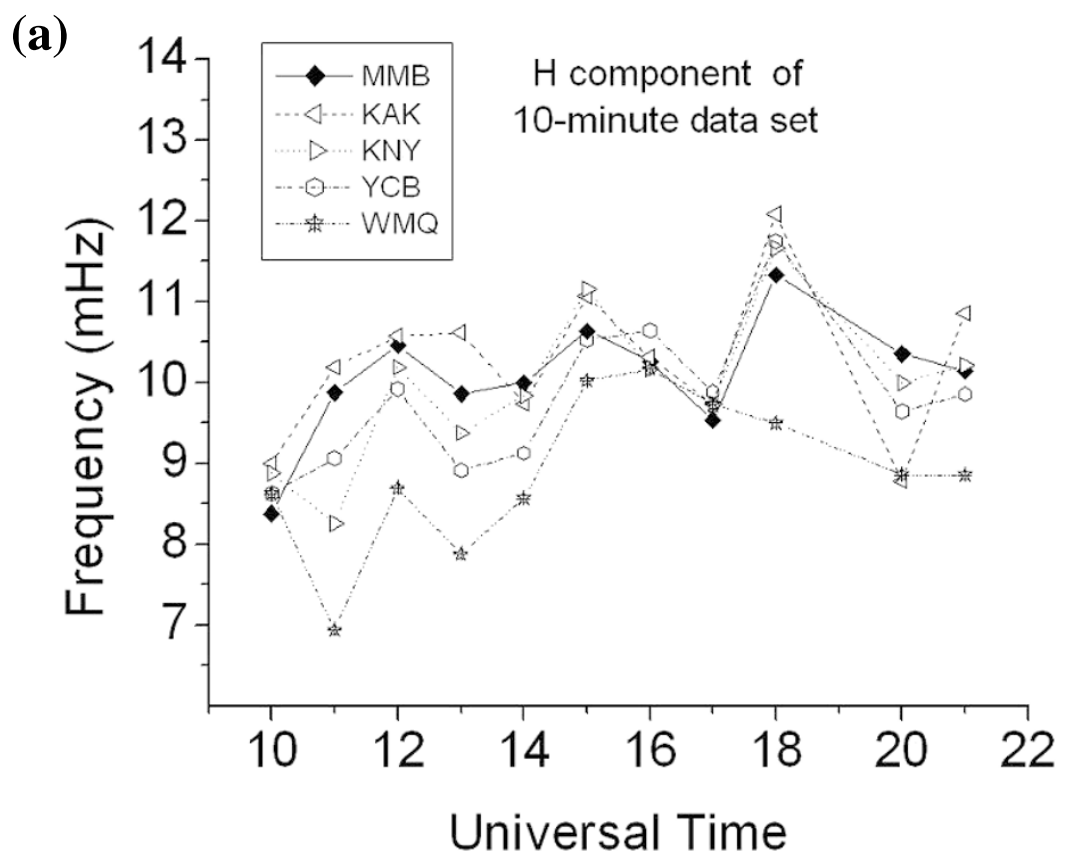

Fig. 4. UT dependence of average frequency. Figures (a), (b), and (c) are average frequencies of the H component for the 10-minute, 5-minute and trailing-5-minute data sets, respectively. Figure (d) shows the average frequency of D component for the 5-minute data set.

\section{Statistical Analysis and Results}

We use a similar method to that used in Kosaka et al. (2002) to get the dominant frequency of Pi2s. That is, the maximum entropy method (MEM) is used to obtain the spectra, and the peak value of each spectrum is taken to be the dominant frequency. The order of the MEM is fixed to 45 for the two 5 minutes data sets and to 60 for the 10-minute data set by referring to the Akaike Information Criterion (Ulrych and Bishop, 1975). Figure 3 gives an example of the spectra which gives the results for the event shown in Fig. 2. The 
(b)

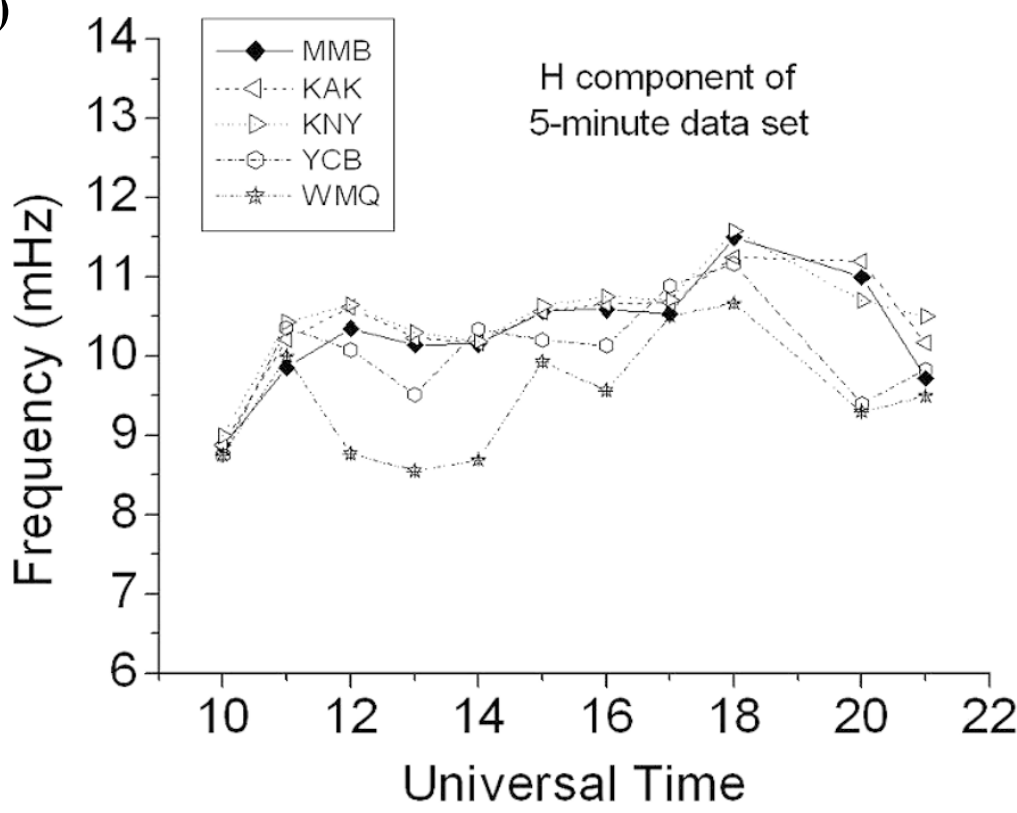

Fig. 4. (continued).

(c)

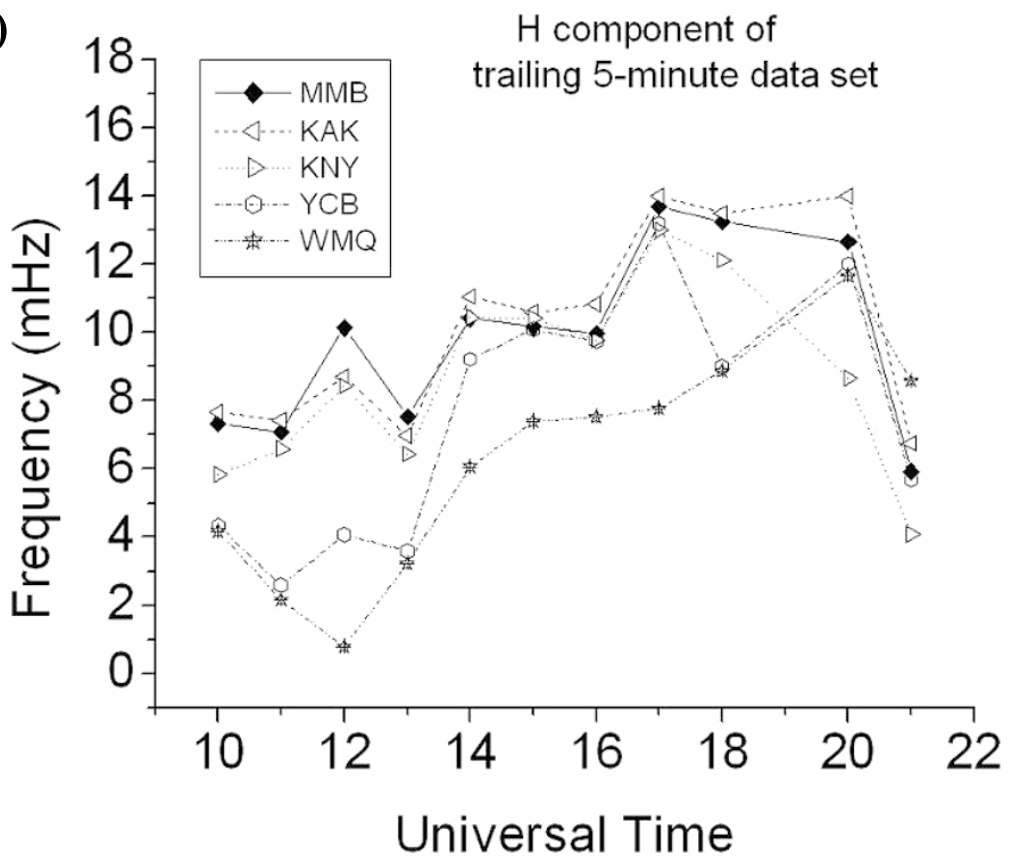

Fig. 4. (continued).

frequency grid is taken to be $0.5 \mathrm{mHz}$ by selecting a proper number of the MEM coefficients (Ulrych and Bishop, 1975).

After obtaining dominant frequencies for each event, we made three kinds of statistical analyses, (1) UT and LT dependence of the dominant frequency for each station, (2) frequency difference between every two stations for each event, and (3) frequency difference between the H and D components for the same event at the same station.

\subsection{UT and LT dependence of dominant frequency}

In order to show the local time dependence, we present the UT and the LT dependence plots of the averaged dominant frequency for the three kinds of data sets in this section. The averaged frequency for each hour was obtained by averaging the frequency of the events which fall into an one-hour bin centered in that hour. We should note that in the UT dependence plot, for different stations, the average frequency of each hour is calculated from the same events recorded simultaneously at those stations, so their difference also shows the longitudinal, namely, local time dependence. In the LT dependence plot, the hourly averaged frequencies are calculated after converting the occurrence time from UT to LT for each event. 
(d)

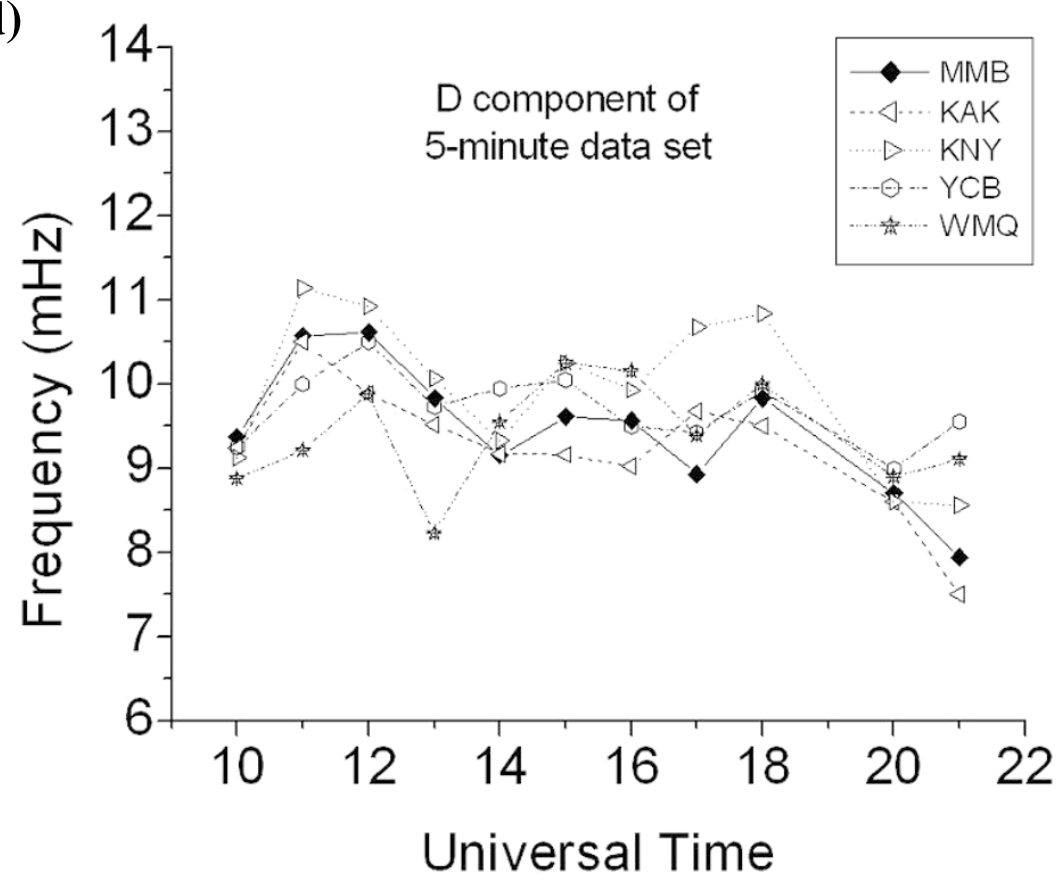

Fig. 4. (continued).

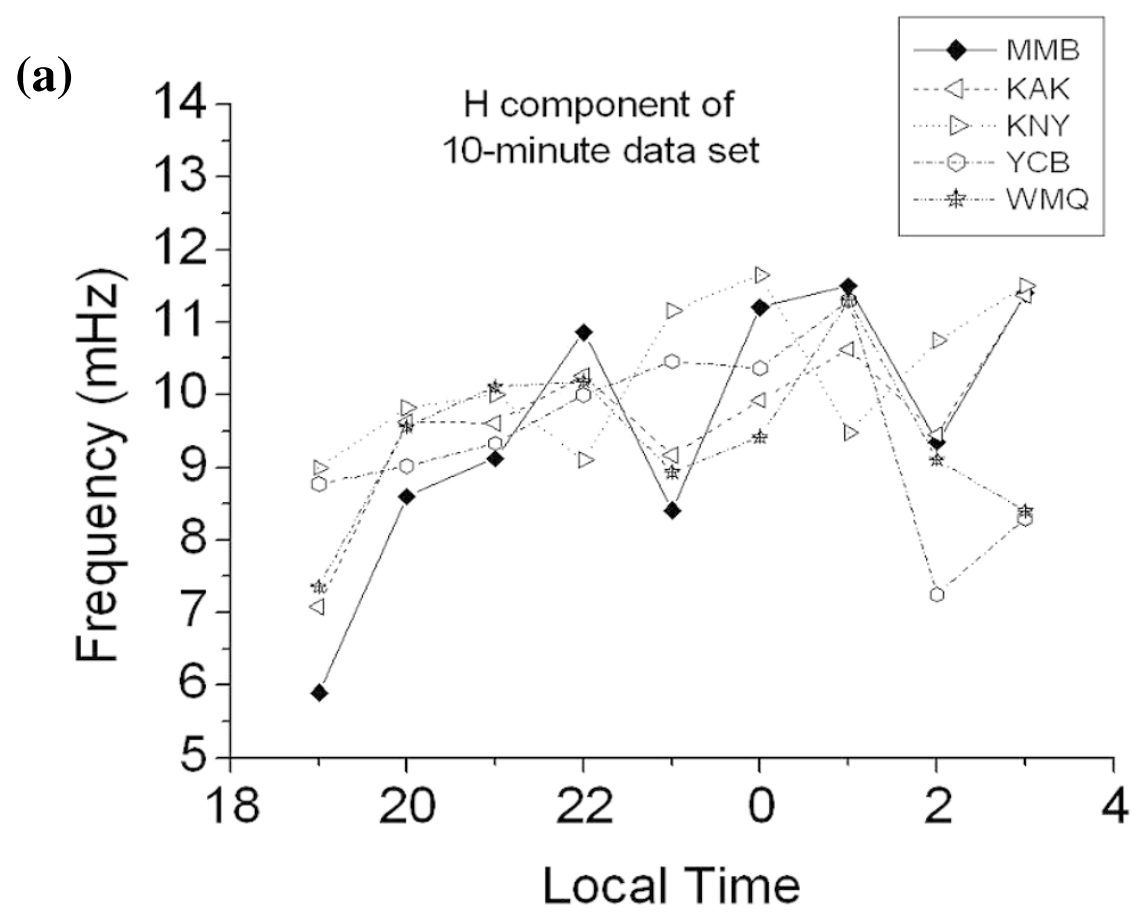

Fig. 5. LT dependence of average frequency. Figures (a), (b) and (c) are average frequencies of the H component for the 10-minute, 5-minute and trailing-5-minute data sets, respectively. Figure (d) shows the results in D component for the 5-minute data set.

Because most of the events fall into 10-21 UT as shown in Fig. 1, we only presented the UT dependence of hourly averaged frequencies for the three data sets during this period and the results were shown in Figs. 4(a), (b), (c) and (d). Considering that there is only one event at $19 \mathrm{UT}$, we ignored the value at 19 UT in the plots. Figs. 4(a), (b) and (c) show the UT dependence of averaged dominant frequency for the $\mathrm{H}$ components for the 10-minute, 5-minute and trailing-5minute data sets, respectively. Figures 4(d) shows the results for the $\mathrm{D}$ component for the 5-minute data set. The results for the $\mathrm{D}$ component for the 10-minute and trailing-5-minute data sets are not shown because their tendency is the same with that for the 5-minute data set.

From Fig. 4, we can find that the average frequencies of western stations are lower than that of eastern stations for the H components from 10 UT to 17 UT. During this period, for the $\mathrm{H}$ component of the three data sets, the variation curves of WMQ always lie in the lowest position, while 
(b)

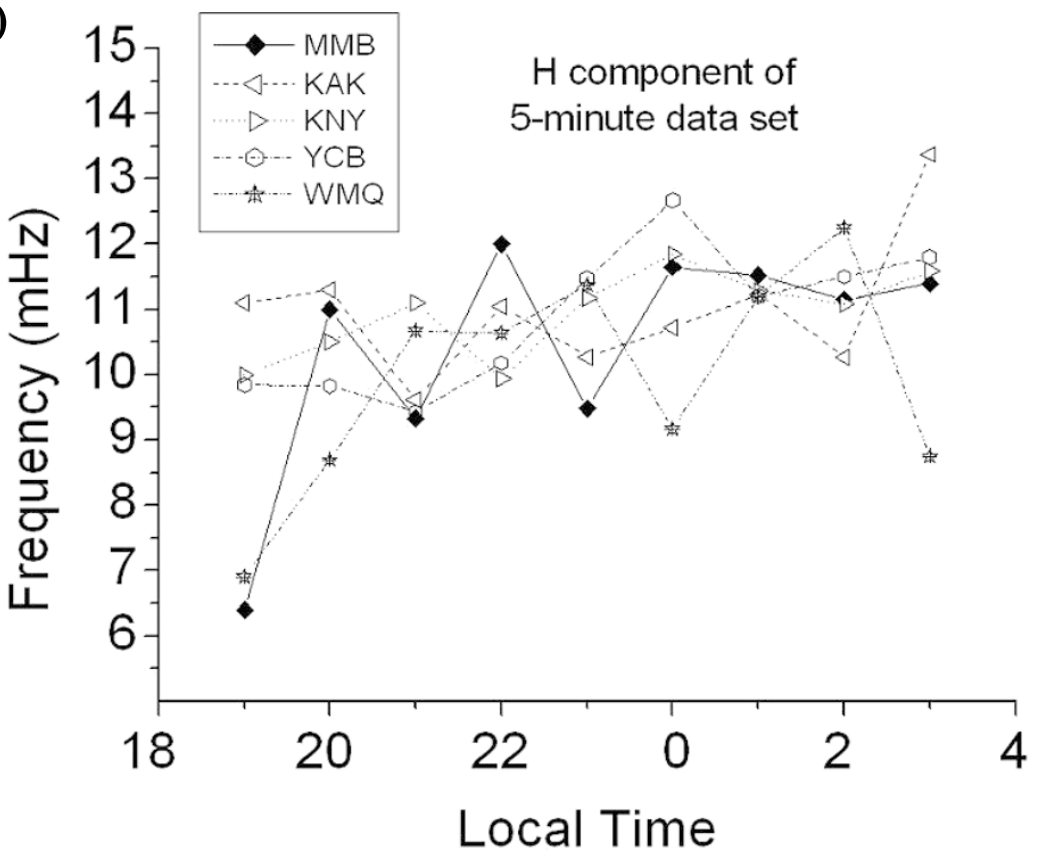

Fig. 5. (continued).

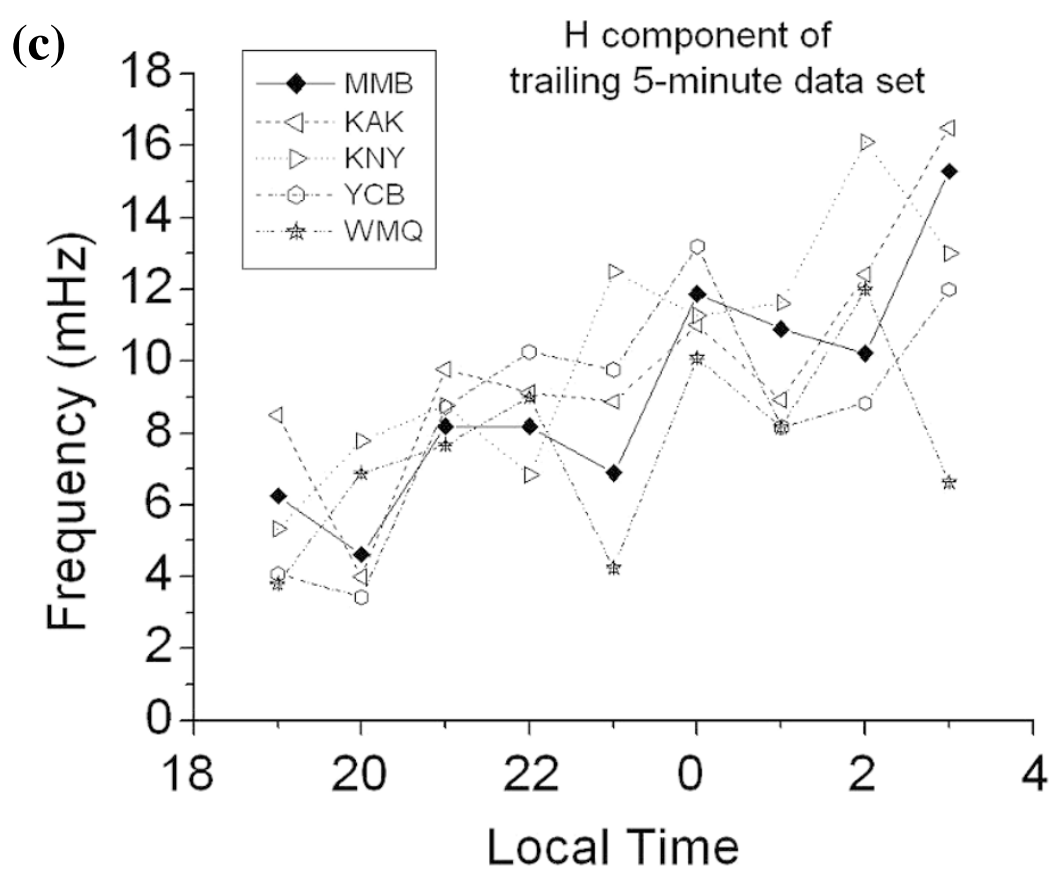

Fig. 5. (continued).

the curves of KAK, KNY and MMB are located on the upper part without much difference and the curves of YCB tends to lie between them. We note that this tendency is not necessarily related to the $L$-value, because the $L$-value at WMQ and MMB are nearly the same. 17 UT is about $02 \mathrm{LT}$ at KAK, KNY and MMB, about $00 \mathrm{LT}$ at YCB and $23 \mathrm{LT}$ at WMQ. From Figs. 4(a) and (b), we can find that the average frequency has a peak at $18 \mathrm{UT}$, which is about $03 \mathrm{LT}$ at KAK, KNY and MMB. Therefore Figs. 4 (a) and (b) suggest that the dominant frequency of Pi2 has LT dependence and has a maximum value at about $03 \mathrm{LT}$.
In order to show the local time dependence of the average frequency more explicitly, we transformed the occurrence time of each event from UT to LT for each station according to the time difference shown in Table 1 and then averaged the dominant frequency within each LT hour. The same procedure is applied to the three data sets and the results are presented in Fig. 5. Figures 5(a), (b) and (c) are averaged dominant frequencies of $\mathrm{H}$ component for the three data sets, respectively. Fig. 5(d) shows the D component for the 5minute data set.

Figures 5 (a), (b) and (c), clearly show that the frequency 
(d)

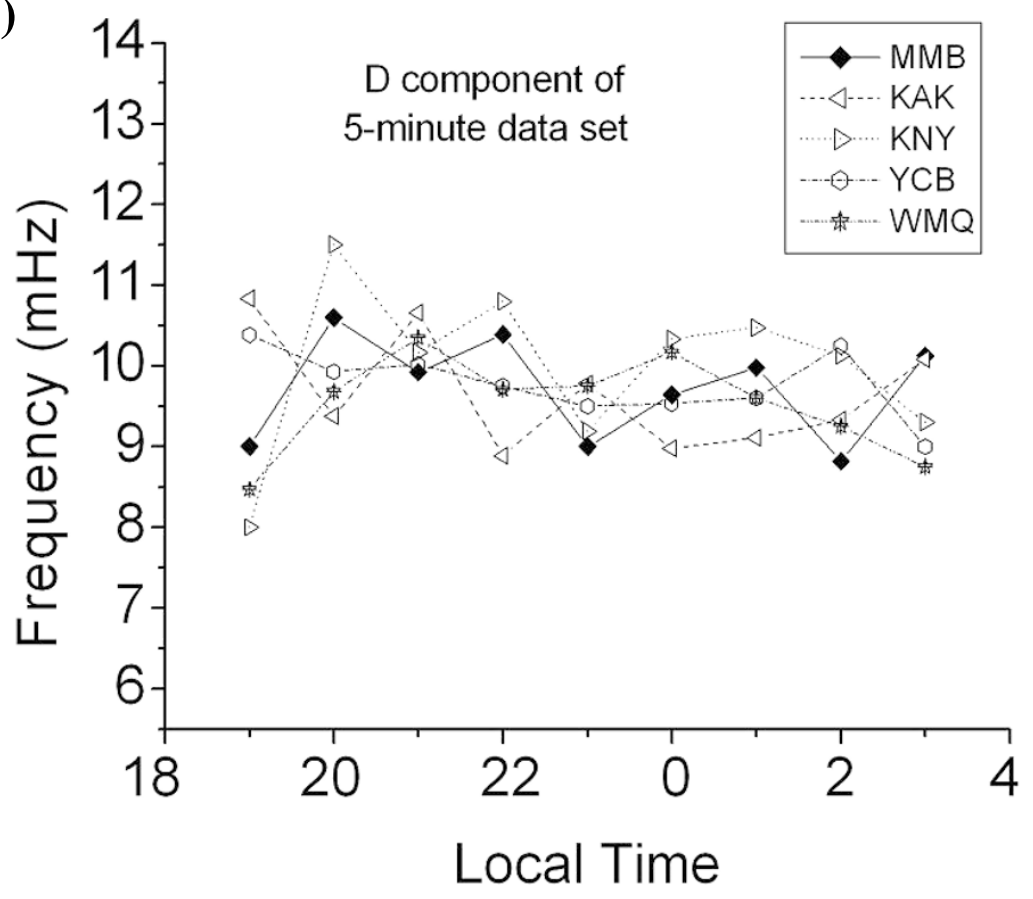

Fig. 5. (continued).

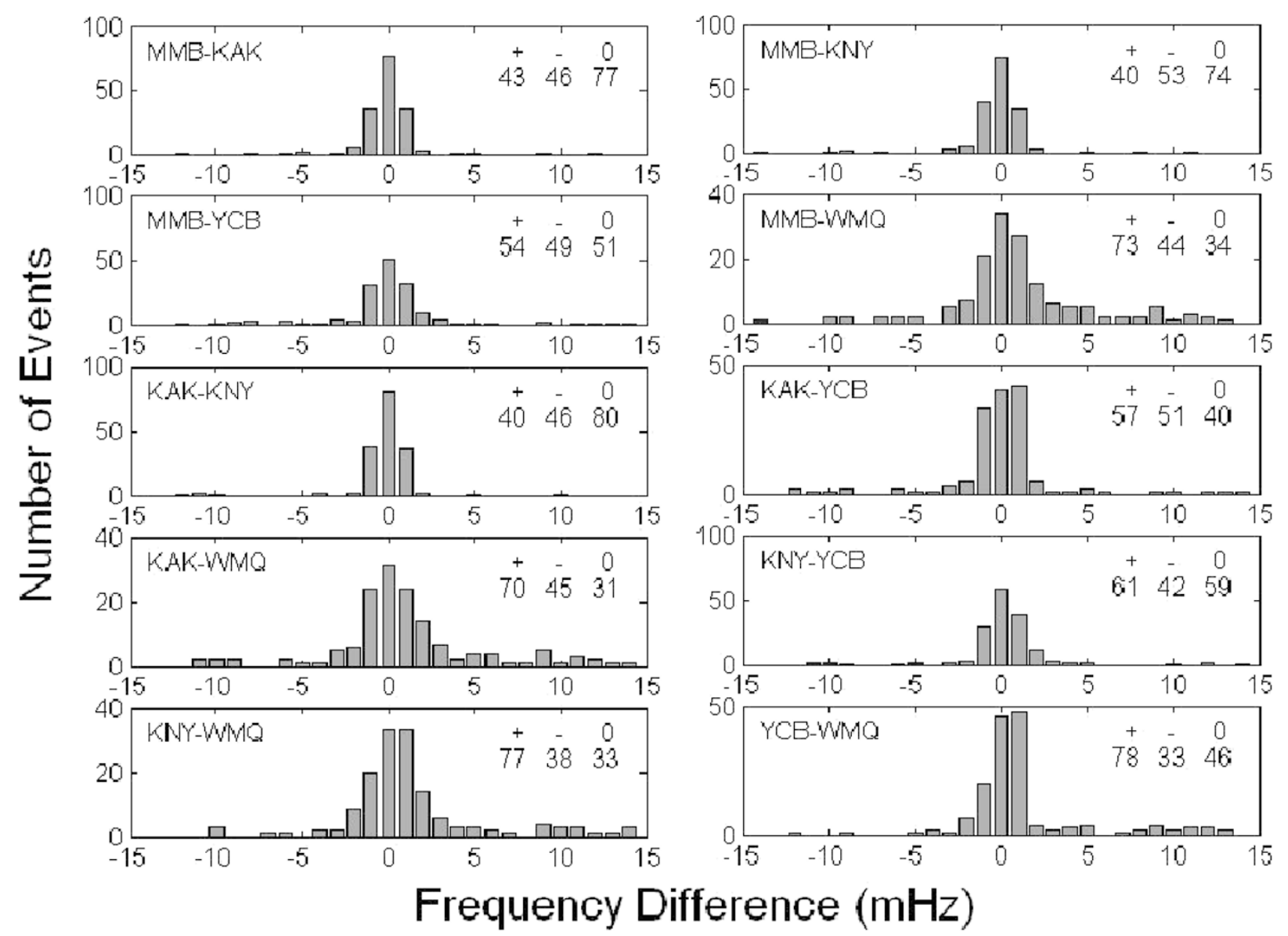

Fig. 6. The distribution of the frequency differences for the H component between every station pairs. The UT range is from 10 UT to 18 UT, which all of the 5 stations are in pre-midnight. The events whose absolute frequency difference is larger than $15 \mathrm{mHz}$ are ignored. The numbers of events which have positive, negative and zero frequency difference are shown on right side of each panel. 


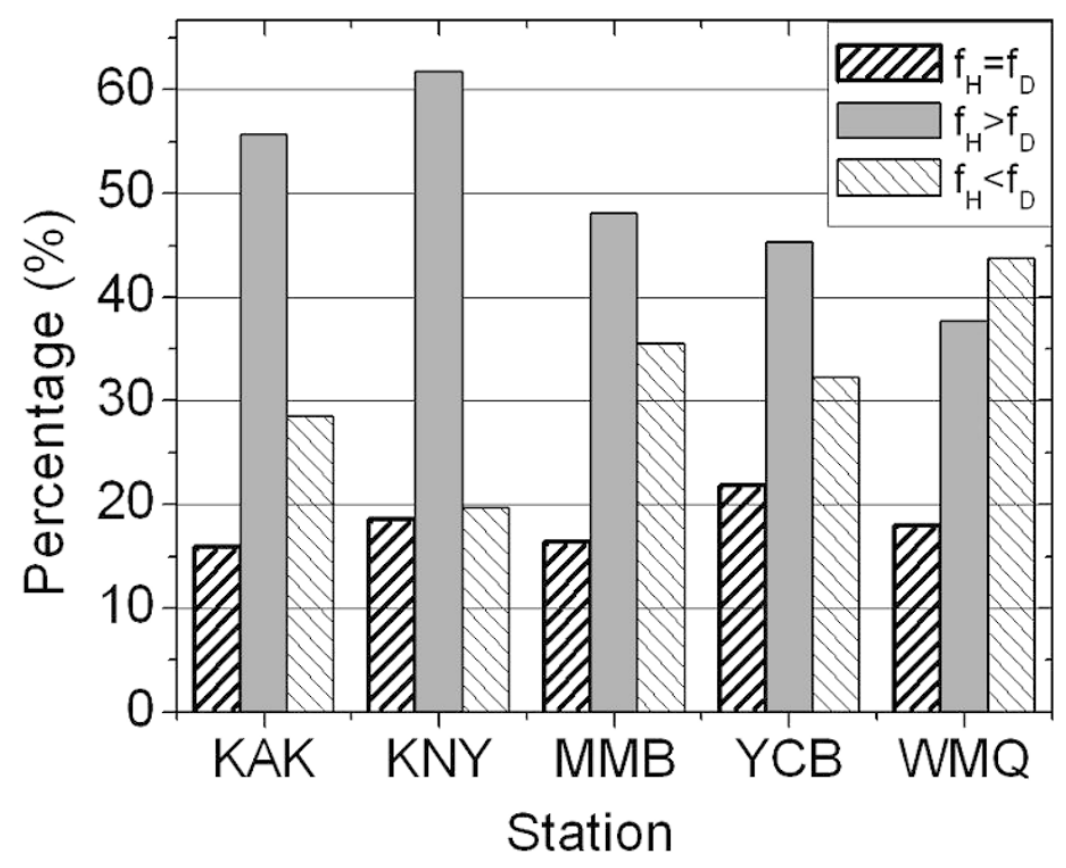

Fig. 7. Frequency difference between the $H$ and $D$ components.

of the $\mathrm{H}$ component increases as the time varies from $19 \mathrm{LT}$ to $03 \mathrm{LT}$. But this tendency is not seen for the $\mathrm{D}$ component as shown in Fig. 4(d) and Fig. 5(d).

These observational results for the 5 low-latitude stations are consistent with what Kosaka et al. (2002) found in the $\mathrm{Pi} 2$ pulsations observed at KAK, that is, the $\mathrm{Pi} 2$ pulsations tend to have higher frequency in post-midnight than that in pre-midnight, and have a peak at 03-04 LT.

By comparing the dominant frequencies of the three data sets, for example, contrasting the results of Figs. 4(a) and (b) with Fig. 4(c) or contrasting the results of Figs. 5(a) and (b) with Fig. 5(c), we should also note that prior to $15 \mathrm{UT}$ in Fig. 4(c) and to 00 LT in Fig. 5(c), the averaged dominant frequency for the trailing-5-minute data set is lower than that for the 10- and 5-minute data sets.

\subsection{Frequency difference for each event and its statisti- cal distribution}

In Fig. 4(a) and (b), the average frequency shows a peak at $18 \mathrm{UT}$, therefore we examined the frequency difference between every two stations for each event occurred from 10 UT to $18 \mathrm{UT}$ and computed their statistical distribution. Because the frequency of the D component does not show any clear LT dependence, only the $\mathrm{H}$ component was used here. The stations ordered from east to west are MMB, KAK, KNY, YCB and WMQ. When we calculate the frequency difference between any two stations, we always subtract the frequency value of western station from that of the eastern one. The statistics of frequency difference is taken in $0.5 \mathrm{mHz}$ resolution, i.e., if the absolute value of the difference is less than $0.5 \mathrm{mHz}$, we take it as zero frequency difference.

In such a way, we investigated the statistical distribution for every station pair for the three data sets. Because the results are similar for the three data sets, we only present the result for the 5-minute data set in Fig. 6. The total number of the events where the frequency difference is positive, negative and zero are indicated on the left side of each panel.
From Figs. 6 we can find that; (1) the frequency difference is nearly zero for a significant part of the events, (2) the number of events with nonzero frequency difference increases as the distance between two stations become longer, (3) there are more events with a positive frequency difference than a negative difference for the cases where the longitudinal distance between two stations is larger, e.g., for MMBYCB, MMB-WMQ, KAK-YCB, KAK-WMQ, KNY-YCB and KNY-WMQ pairs.

\subsection{Frequency difference between the $H$ and $D$ compo- nents}

For understanding the reason why the frequency of D component has no LT dependence, we investigated the frequency difference between $\mathrm{H}$ and $\mathrm{D}$ for the same event at a given station. The results are presented in Fig. 7. We can find that no more than $20 \%$ of the events have identical frequency for both $\mathrm{H}$ and $\mathrm{D}$ components, and the frequency of the $\mathrm{H}$ component is generally higher than that of the D component.

\subsection{Phase difference between MMB and WMQ}

We choose MMB and WMQ, which are in nearly the same latitude but have the largest distance among the 5 stations, to present the phase difference properties for the 183 events for both the $\mathrm{H}$ and $\mathrm{D}$ components. Figure 8 shows that the phase differences concentrate around zero for the $\mathrm{H}$ component, but they are much scattered on both sides of the zero for the D component.

\section{Summary and Discussion}

The 183 Pi2 pulsations simultaneously recorded at the 5 stations located in low-latitudes were used to statistically analyze the frequency properties. By analyzing the LT dependence of the dominant frequency for the three kinds of data sets, we confirmed that the dominant frequency of the $\mathrm{H}$ component are lower on the dusk side than that on the dawn side, and the dominant frequencies have a maximum value at 


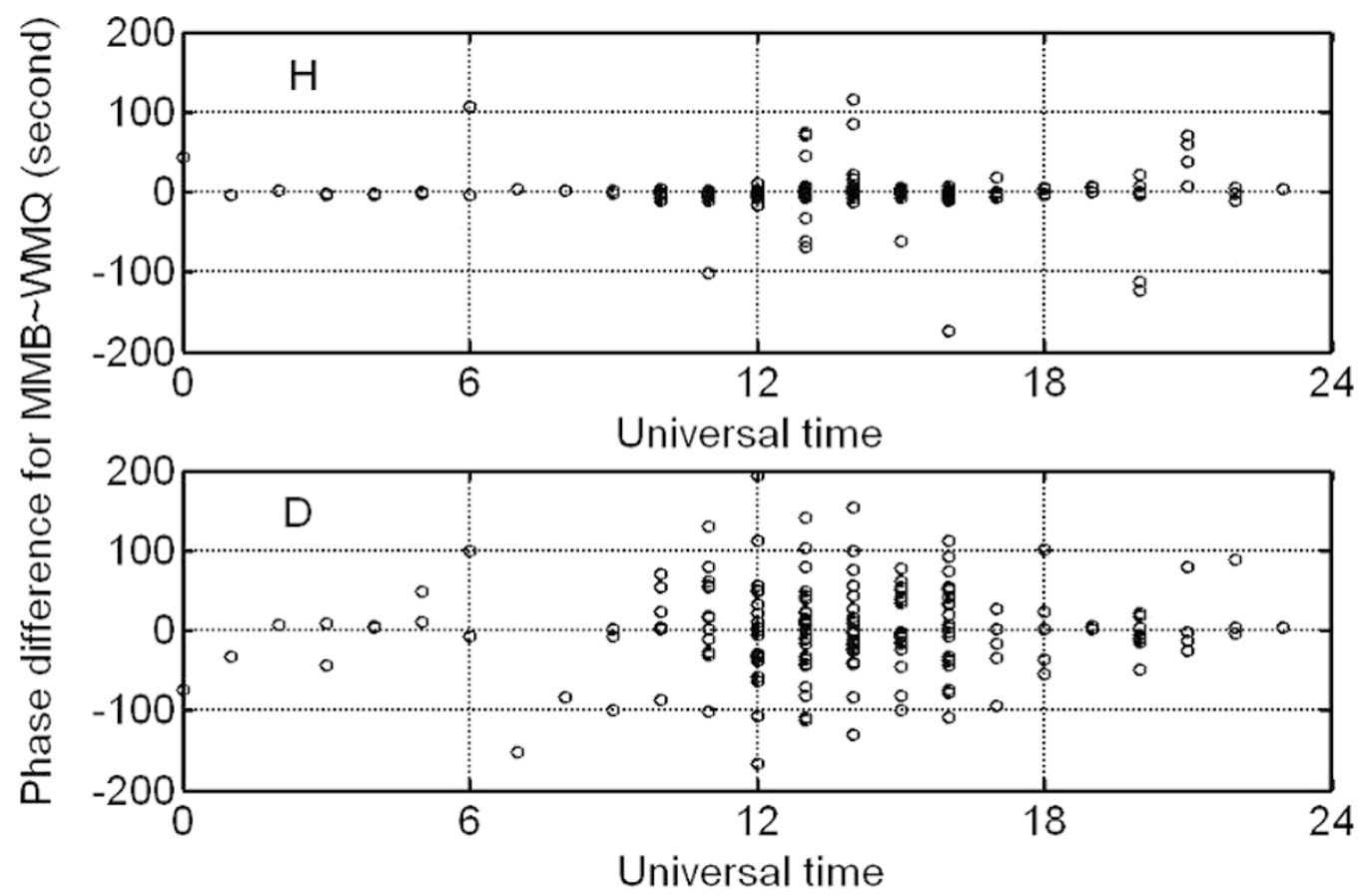

Fig. 8. Phase difference between MMB and WMQ for the H and D components.

03 LT. However, the D component does not show such LT dependence.

To understand the LT dependence of the frequency of Pi2 waves, we need to consider the following two aspects. Firstly, in recent years, many researchers, such as Sutcliffe and Yumoto (1989), Takahashi et al. (1995, 2003), Lee (1996), Cheng et al. (2000) and Denton et al. (2002), suggested that a cavity resonance mode of the plasmasphere is a reasonable mechanism for the generation of $\mathrm{Pi} 2$ pulsations within the plasmapause at low latitudes. Nosé et al. (2003) found that the plasmaspheric cavity mode resonance can be established even on the morning side. Although the cavity mode oscillations have properties that the spatial extent is very large and the oscillation frequencies are the same throughout the cavity as summarized by Keiling et al. (2001), Fujita et al. (2003) demonstrated that the cavity resonance mode can have a local time dependence of the frequency when the plasmasphere has a non-uniform structure in longitudinal direction. Takahashi et al. (2003) also discussed that as the size of the plasmasphere increases, the cavity mode frequency decreases as a result of the increase in the fast mode bounce time between the radial boundaries. That is, considering the non-uniform structure of plasmasphere, the cavity mode could also cause the LT dependence of the dominant frequency of Pi2s, which is consistent with the observational results presented in this paper.

Secondly, if the surface wave at the plasmapause is the dominant mechanism of $\mathrm{Pi} 2$ pulsations, the shape and the size of the plasmasphere can affect the period of the wave. The plasmasphere bulging out to the dusk side (Carpenter, 1966) gives a possibility to cause the local time dependence of the frequency. Using the Global Core Plasma Model (GCPM) (Gallagher et al., 2000), Kosaka et al. (2002) estimated the oscillation frequency of the surface wave for different geomagnetic activities, which correspond to different shapes of the plasmasphere. Their model calculation shows that the surface wave frequency increases as the local time changes from 18 LT to $04 \mathrm{LT}$, which is also consistent with the observational results obtained in this paper. They suggest that the surface wave mode is a possible generation mechanism for mid- and even low-latitude Pi2s.

Figures 6 show that the number of events with frequency difference less than $1 \mathrm{mHz}$ is the majority for each station pair, and the number of events with a positive frequency difference is usually larger than that of negative frequency difference for the $\mathrm{H}$ component except for the cases between the three Japanese stations which locate in narrow longitudinal width. For explaining this fact, we suggest that there are two types of Pi2s among the 183 events, i.e., one is centered at zero frequency difference and another has a slight shift toward positive direction. That is, one has a constant frequency throughout the local time and another has LT dependence. The superposition of the two types of events results in the LT dependence of the frequency statistically. If this is the case, we suggest that the constant (or nearly equal) frequency events are caused by the cavity mode because the frequency is expected to be the same throughout the cavity in general.

For the LT dependent events, we suggest that they likely belong to either one of the two possible modes. One is the cavity mode as suggested by Fujita and Itonaga (2003), that is, the longitudinal non-uniform structure of the plasmaphere could cause the difference of the cavity mode frequency and could be the reason of the LT dependence of the frequency. Another is the surface wave mode as suggested by Kosaka et al. (2002).

Kosaka et al. (2002) showed that the LT dependence of the frequency is obvious when $\mathrm{Kp}$ index is higher, but not clear when it is lower. When the Kp is higher, the plasmasphere shrinks and the foot-point of the plasmapause moves 
toward the low latitudes and the waves could be observed in this area, so we also do not rule out the possibility that the low-latitude Pi2s could be caused by the surface wave mode although the surface waves are localized to the plasmapause and thought to be difficult to propagate to low-latitudes. We should note that the Kp index is 4 for the event shown in Fig. 2, where the dominant frequency has obvious LT dependence and the phase shift is also clear.

From the ground-based observations alone, we can not confirm whether both of the two modes coexist or only one of them is dominant for causing the LT dependence in frequency. To confirm it, a contrast analysis between ground and satellite observations is necessary.

A possible explanation for the distribution of the frequency difference spreading to both sides is that the variance in frequency is caused by the variability in the longitudinal asymmetry of the plasmasphere. The events with constant frequency in LT occur when the plasmasphere has a symmetric structure, while the LT dependent events occur when the plasmasphere bulges out to dusk side or distorted in various way. It is necessary to increase the number of events to conclude that the distribution is a superposition of two different distributions or single distribution.

Figure 7 shows that only about $20 \%$ of events have identical frequency between $\mathrm{H}$ and $\mathrm{D}$ components. Keiling et al. (2001) and Lester and Orr (1983) also reported the cases of Pi2s where the H and D were decoupled. So we suggest that the $\mathrm{H}$ and $\mathrm{D}$ components of Pi2s observed on the ground can be excited independently, for example, the D component might have more intimate relation with substorm current system than the H component does. Lester et al. $(1983,1984)$ studied the polarization and phase properties for mid-latitude Pi2 pulsations. Their results suggested that the substorm current wedge controls the sense of horizontal polarization as well as the phase difference between stations pairs. Recently, Fujita et al. (2002) made a numerical simulation of the Pi2 pulsations associated with the subsorm current wedge and obtained consistent results with the observations by Lester et al. (1983, 1984). According to our results as shown in Fig. 8, the phase differences between east-west stations pairs are negligible for the $\mathrm{H}$ component but are clear for the $\mathrm{D}$ component. So it is plausible to consider that the H component is due to the cavity mode with in phase resonance in the whole cavity, but the D component is caused by a different mechanism which shows propagation properties.

Another point worth to be noted is that the average frequency of trailing-5-minute data set are lower than that of the other two data sets in pre-midnight region (see Figs. 4 and 5). This means that the frequency tend to decrease as the oscillation damps, or there exist two modes and one of them with lower frequency lasts for longer period.

\section{Conclusion}

The dominant frequency of the Pi2 pulsations observed at 5 stations at low-latitudes was examined statistically. The followings are our main results.

1) It was confirmed that the dominant frequencies of the $\mathrm{H}$-component of low-latitude $\mathrm{Pi} 2$ pulsations have LT dependence, i.e., they increase from 19 LT to 03 LT.
2) The D component does not show any clear LT dependence, and only about $20 \%$ of events have identical frequency for both $\mathrm{H}$ and $\mathrm{D}$ components, which suggest that the $\mathrm{H}$ and $\mathrm{D}$ components of $\mathrm{Pi} 2 \mathrm{~s}$ are excited independently.

3) A part of the Pi2s observed at low-latitudes show no frequency differences in the local time examined and could be the cavity mode. The remaining part of the events, which have the LT dependence in frequency, also might result from the cavity mode, but we also do not rule out the possibility of the surface wave mode.

4) A possible explanation for the spread distribution of the frequency difference could be that it is caused by the variability in longitudinal structure of the plasmasphere.

5) For some events, the dominant frequency of Pi2 pulsations decreases as the Pi2 oscillation damps.

Acknowledgments. The KAK, KNY and MMB data are provided by Kakioka Magnetic Observatory. The WMQ and YCB data are observed by Urumqi Basic Seismic Station and Yinchuan Magnetic Observatory of China in collaboration with China Seismological Bureau and Kyoto University. We thank K. Kosaka for his help and discussion in preparing the data sets. This study has been supported in part by grant 13640451 under Japan Society for Promotion of Science (JSPS) and by grant from Heiwa Nakajima Foundation.

\section{References}

Allan, W., E. M. Poulter, and S. P. White, Hydromagnetic wave coupling in the magnetosphere - plasmapause effects on impulse-excited resonances, Planet. Space Sci., 34, 1189-1200, 1986.

Carpenter, D. L., Whistler studies of the plasmapause in the magnetosphere, 1 , Temporal variations in the position of the knee and some evidence on the plasma motions on near knee, J. Geophys. Res., 71, 693-709, 1966.

Chen, L. and A. Hasegawa, A theory of long-period magnetic pulsations, 2. Impulse excitation of surface eigen-mode, J. Geophys. Res., 79, 1033, $1974 \mathrm{~b}$.

Cheng, C.-C., J.-K. Chao, and K. Yumoto, Spectral power of low-latitude $\mathrm{Pi} 2$ pulsations at the $210^{\circ}$ magnetic meridian stations and plasmaspheric cavity resonances, Earth Planets Space, 52, 615-627, 2000.

Dento, R. E., D.-H. Lee, K. Takahashi, J. Goldstein, and R. Anderson, Quantitative test of the cavity resonance explanation of plasmaspheric PI2 frequencies, J. Geophys. Res., 107(A7), 10.1029/2001JA000272, 2002.

Fujita, S and M. Itonaga, A plasmaspheric virtual resonance in a longitudinally non-uniform plasmasphere, Earth Planets Space, 55, 219-222, 2003.

Fujita, S., H. Nakata, M. Itonaga, A. Yoshikawa, and T. Mizuta, A numerical simulation of the Pi2 pulsations associated with the substorm current wedge, J. Geophys. Res., 107, 10.1029/2001JA900137, 2002.

Gallagher, D. L., P. D. Craven, and R. H. Comfort, Global core plasma model, J. Geophys. Res., 105, 10819-10833, 2000.

Keiling, A., J. R. Wygant, C. Cattel, K.-H. Kim, C. T. Russell, D. K. Milling, M. Temerin, F. S. Mozer, and C. A. Kletzing, Pi2 pulsations observed with the Polar satellite and ground stations: Coupling of trapped and propagating fast mode waves to a midlatitude field line resonance, $J$. Geophys. Res, 106, 25891-25904, 2001.

Kosaka, K., T. Iyemori, M. Nosé, M. Bitterly, and J. Bitterly, Local time dependence of the dominant frequency of $\mathrm{Pi} 2$ pulsations at mid- and lowlatitudes. Earth Planets Space, 54, 771-781, 2002.

Lanzerotti, L. J. and L. Medford, Local night, impulsive (Pi2 type) dydromagnetic wave polarization at low latitudes, Planet. Space Sci., 32, 135, 1984.

Lee, D.-H., Dynamics of MHD wave propagation in the low-latitude magnetosphere, J. Geophys. Res., 101, 15371-15386, 1996.

Lee, D.-H. and R. Lysak, MHD waves in a three-dimensional dipolar magnetic field: a search for Pi2 pulsations, J. Geophys. Res., 104, 28691$28699,1999$. 
Lester, M. and D. Orr, Correlations between ground observations of Pi2 geomagnetic pulsations and satellite plasma density observations, Planet. Space Sci., 31, 143, 1989.

Lester, M., W. J. Hughes, and H. J. Singer, Polarization patterns of Pi2 magnetic pulsations and the substorm current wedge, J. Geophys. Res., 88, 7958-7966, 1983.

Lester, M., W. J. Hughes, and H. J. Singer, Longitudinal structure in Pi2 pulsation and the substorm current wedge, J. Geophys. Res., 89, 54895494, 1984.

Nosé, M., T. Iyemori, M. Takeda, T. Kamei, D. K. Milling, D. Orr, H. J. Singer, E. W. Worthington, and N. Sumitomo, Automated detection of Pi2 pulsations using wavelet analysis: 1 . Method and an application for substorm monitoring, Earth Planets Space, 50, 773-783, 1998.

Nosé, M., K. Takahashi, T. Uozumi, K. Yumoto, Y. Miyoshi, A. Morioka, D. K. Milling, P. R. Sutcliffe, H. Matsumoto, T. Goka, and H Nakata, Multipoint observations of a $\mathrm{Pi} 2$ pulsation on morning side: The 20 September 1995 event, J. Geophys. Res., 108(A5), 1219, doi 10.1029/2002Ja009747, 2003.

Olson, J. V., Pi2 pulsations and substorm onsets: A review, J. Geophys. Res., 104, 17499-17520, 1999.

Sutcliffe, P. R., The association of harmonics in Pi2 power spectra with the plasmapause, Planet. Space Sci., 23, 1581-1587, 1975.
Sutcliffe, P. R. and K. Yumoto, Dayside Pi2 pulsations at low latitudes, Geophys. Res. Lett., 16, 887-890, 1989.

Sutcliffe, P. R. and K. Yumoto, On the cavity mode nature of low latitude Pi2 pulsations, J. Geophys. Res., 96, 1543-1551, 1991.

Takahashi, K., S.-i. Ohtani, and B. J. Anderson, Statistical analysis of Pi 2 pulsations observed by the AMPTE CCE spacecraft in the inner magnetosphere, J. Geophys. Res., 100, 21929-21941, 1995.

Takahashi K., D.-H. Lee, M. Nose, R. R. Anderson, W. J. Hughes, CRRES electric field study of the radial mode structure of Pi2 pulsations, $J$. Geophys. Res., 108(A5), 1210, doi:10.1029/2002JA009761, 2003.

Ulrych, T. J. and T. N. Bishop, Maximum entropy spectral analysis and autoregressive decomposition, Reviews of Geophysics and Space Physics, 13, 183-200, 1975.

Yeoman, T. K. and D. Orr, Phase and spectral power of mid-latitude Pi2 pulsations: Evidence for a plasmaspheric cavity resonance, Planet. Space Sci., 37, 1367-1383, 1989.

D. Han (e-mail: hands@kugi.kyoto-u.ac.jp), T. Iyemori, Y. Gao, Y. Sano, F. Yang, W. Li, M. Nosé 